\title{
Papulonodular secondary syphilis: a rare clinic presentation con- firmed by serologic and histologic exams ${ }^{*}$
}

\author{
John Verrinder Veasey ${ }^{1}$ \\ Maria Fernanda Feitosa de Camargo Boin ${ }^{2}$ \\ Jessica Chia Sin Chen ${ }^{3}$
}

\author{
Rute Facchini Lellis ${ }^{1}$ \\ Pedro Loureiro Porto ${ }^{3}$
}

DOI: http:/ /dx.doi.org/10.1590/abd1806-4841.20163958

\begin{abstract}
Syphilis is a sexually transmitted disease caused by Treponema pallidum and divided into three stages according to the duration of the disease: primary, secondary and tertiary. Secondary syphilis has diverse clinical presentations, such as papular-nodular lesions. This presentation is rare, with 15 cases reported in the literature over the past 20 years. We report a case of secondary syphilis with papular-nodular lesions in a healthy 63-yearold patient, who has presented treponema in immunohistochemical examination of the skin lesions.
\end{abstract}

Keywords: Histology; Syphilis; Syphilis, cutaneous; Treponema; Treponemal infections; Treponema pallidum

\section{INTRODUCTION}

Syphilis (LUES) is a sexually transmitted disease (STD) caused by Treponema pallidum bacteria and transmitted mainly by sexual contact. ${ }^{1}$

The natural history of this disease shows the evolution which has alternating periods of activity with clinical, immunologic and histopathologic distinct characteristics (primary, secondary and tertiary syphilis) and latency periods (latent syphilis). ${ }^{2}$ The secondary stage is accompanied by polyadenomegaly and polymorphism of cutaneous lesions. There are reports of macular, papular, follicular, lichenoid, psoriasiform and corymbiform clinical presentations, among others. ${ }^{3}$ The presentation of secondary syphilis with papulo-nodular lesions is rare, with 15 cases reported in the literature over the past 20 years.

As happens in the clinic, the histopathological examination is also polymorphic without pathogenic patterns. The determination of the agent would be then the diagnostic confirmation of the disease. However, its visualization by routine staining (hematology and eosin) presents obstacles, which makes additional methods such as immunohistochemical examination necessary. ${ }^{4,5}$

\section{CASE REPORT}

Male patient, 63 years old, married, born and raised in São Paulo/SP, with a history of lesions disseminated over his body for one month. Furthermore, he had lost $12 \mathrm{~kg}$ in two months and denied other commodities. He had used loratadine, invermectin and topical betamethasone with no improvement.

The physical examination revealed dermatosis disseminated all over his body, characterized by erythematous macules of 0.5 to $3 \mathrm{~cm}$ in diameter and infiltrated erythematous papules, mostly on his back (Figure 1). The penis showed erythematous macules and a well-delimited ulcer of $1 \mathrm{~cm}$ with a clean erythematous background and marked borders, which according to the patient was in a healing process, in addition to painless, fibroelastic occipital adenomegaly of $1.5 \mathrm{~cm}$ in diameter (Figure 2).

Based on the clinical picture, the hypothesis of secondary syphilis was raised. Serologys were requested and one lesion on the back was biopsied. Serologic reactions for syphilis showed VDRL 1/512 and positive FTA-ABS. Serology for HIV, hepatitis B and $C$ were negative; HIV test was repeated and remained negative. Histopathological examination by

Received on 18.08.2014

Approved by the Advisory Board and accepted for publication on 09.12.2014

Work performed at Hospital da Santa Casa de São Paulo - São Paulo (SP), Brazil.

Financial Support: None.

Conflict of Interests: None.

Santa Casa de Misericórdia de São Paulo - São Paulo (SP), Brazil.

Private Practice - Londrina (PR), Brazil.

Faculdade de Ciências Médicas da Santa Casa de São Paulo (FCMSC-SP) - São Paulo (SP), Brazil.

(C)2016 by Anais Brasileiros de Dermatologia 
HE revealed deep and superficial perianexial and perivascular lichenoid interface dermatitis with predominance of plasmocytes with perineuritis. Immunohistochemical examination was performed using a specific polyclonal antibody for T. pallidum, which revealed the presence of spirochetes (Figure 3).

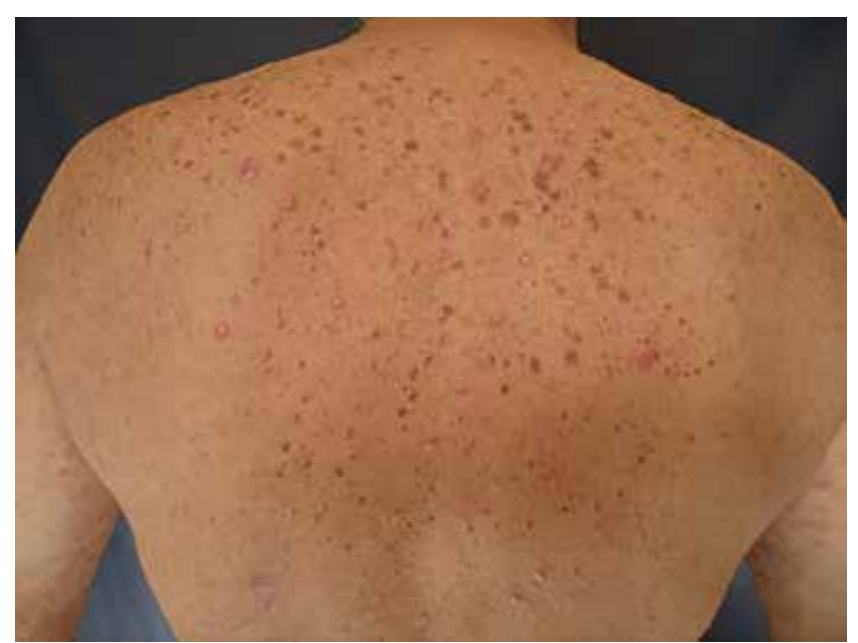

FIGURE 1: Disseminated dermatosis on the back characterized by well-delimited infiltrated papules

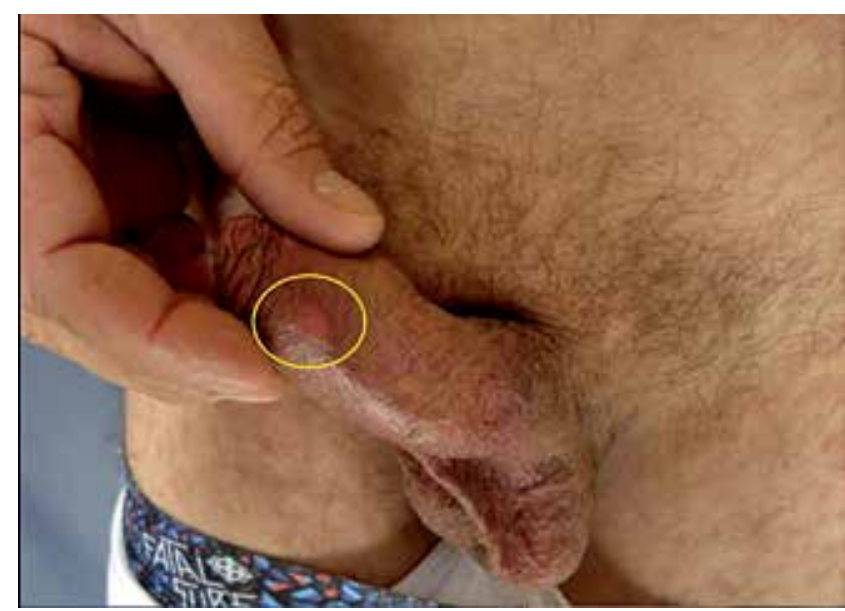

FiguRE 2: Dermatosis located on the penis characterized by an ulcer of clear background and well-delimited borders (yellow circle)
Diagnosis of secondary syphilis by clinical-serological-histological correlation was then verified.

\section{DISCUSSION}

Secondary syphilis manifests itself through diverse clinical presentations, thus it is known as the "great imitator". Secondary lesions appear 6 to 8 weeks after inoculation of Treponema. The most observed clinical presentation in secondary syphilis is the syphilitic roseola with rare cases described as the "papulo-nodular" form. ${ }^{1}$ As in the case presented, nodular syphilis is characterized by multiple erythematous-violaceous plaques and nodules that may affect palms and soles, besides lesions in mucosae. Differential diagnosis includes inflammatory and infectious diseases and even nonproliferation processes such as sarcoidosis, cutaneous tuberculosis, leukemia and lymphoma. In the case presented, the patient was in a transition phase between the end of the primary lesion healing on the penis and the onset of secondary stage with disseminated lesions.

The diagnosis of syphilis and the choice of complementary tests must be based on the evolutive phase of the disease. In case of chancre and condyloma lata diagnosis may be achieved by demonstrating Treponema obtained by analysis of skin scratches from lesions. ${ }^{2}$ The use of serology can be done from second or third week after onset of the chancre, when antibodies can be detected. In serology two types of tests are used: non-specific, antilipidemic or reaginic and specific or antitreponemic. In secondary syphilis both tests are positive.

The demonstration of Treponema in the back lesion corroborates the systemic dissemination of the agent. The presence of Treponema was already validated in both the condyloma lata and syphilids. ${ }^{4}$ However, only condyloma lata is considered infective due to its friable consistency.

Histological examinations of secondary lesions are as diverse as the clinical ones. The predominant histological pattern may be hyperkeratotic, psoriasiform or ulcerated. In general the dermis presents a

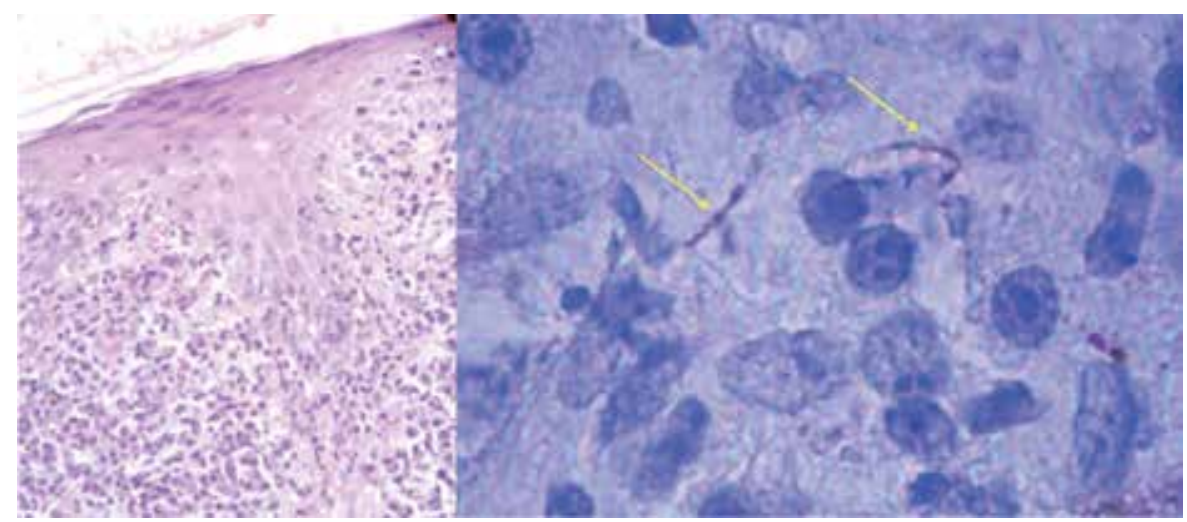

Figure 3: Histological examination by HE staining $(x 400)$ presenting a pattern of interface and plasmocytes. Immunohistochemistry (immersion, x1000) showing treponemas (yellow arrows) 
superficial and deep perivascular infiltrate permeated by plasmocytes. In late secondary lesions epithelioid granulomas are shown. ${ }^{4}$

Gemma Martin et al demonstrated in a study with 34 patients, 8 being primary syphilis and $26 \mathrm{sec}-$ ondary syphilis, the presence of Treponema in eight cases of primary and in 21 cases of secondary, through immunohistochemistry, and in none of these cases the presentation was papulo-nodular. ${ }^{4}$
Our patient was treated with a weekly dose of intramuscular Benzathine penicillin 2,400,000 IU for 2 weeks, with progressive improvement of symptoms until complete remission of lesions.

In conclusion, diagnosing a patient with unusual manifestations of secondary syphilis may be difficult and the differential with other papulo-nodular lesions must be established. The possibility of syphilis should be considered in cases of disseminated dermatosis due to their high incidence in our midst and the variety of clinical presentations in the secondary type.]

\section{REFERENCES}

1. Belda Junior W, Di Chiacchio N, Criado PR. Sífilis adquirida e Congênita. In: Belda Junior W, Di Chiacchio N, Criado PR, orgs. Tratado de Dermatologia. 1. ed. São Paulo: Atheneu; 2010. p. 1257-1278.

2. Avelleira JCR, Bottino G. Syphilis: diagnosis, treatment and control. An Bras Dermatol, 2006; 81:111-26.

3. Baughn RE, Musher DM. Secondary syphilitic lesions. Clin Microbiol Rev. 2005;18:205-16.

4. Martín-Ezquerra G, Fernandez-Casado A, Barco D, Jucglà A, Juanpere-Rodero N, Manresa JM, et al. Treponema pallidum distribution patterns in mucocutaneous lesions of primary and secondary syphilis: an immunohistochemical and ultrastructural study. Hum Pathol. 2009;40:624-30.

5. Buffet M, Grange PA, Gerhardt P, Carlotti A, Calvez V, Bianchi A, et al. Diagnosing Treponema pallidum in Secondary Syphilis by PCR and Immunohistochemistry. J Invest Dermatol. 2007;127:2345-50.
MAILING ADDRESS:

John Verrinder Veasey

Rua Dr Cesário Mota Junior, 112

01221-020 - Vila Buarque - São Paulo -SP

Brazil

E-mail:johnveasey@uol.com.br

How to cite this article: Veasey JV, Lellis RF, Boin MFFC, Porto PL, Chen JCS. Papulo-nodular secondary syphilis: a rare clinic presentation confirmed by serologic and histologic exams. An Bras Dermatol. 2016;91(2):205-7. 